\title{
Exploring Interruption in HRI using Wizard of Oz
}

\author{
Paul Saulnier, Ehud Sharlin, Saul Greenberg \\ Department of Computer Science, University of Calgary \\ Calgary, Canada \\ p.saulnier@ucalgary.ca, ehud@cpsc.ucalgary.ca, saul.greenberg@ucalgary.ca
}

\begin{abstract}
We are interested in exploring how robots controlled using Wizard of $\mathrm{Oz}(\mathrm{WoO})$ should interrupt humans in various social settings. While there is considerable work on interruption and interruptibility in HCI, little has been done to explore how these concepts will map robotic interaction. As part of our efforts to investigate interruption and interruptibility in HRI we used WoO-based methodology to investigate robot behaviours in a simple interruption scenario. In this report we contribute a design critique that discusses this methodology, and common concerns that could be generalized to other social HRI experiments as well as reflections on our future interruption HRI research
\end{abstract}

Keywords-component; Social, human-robot interaction, methodology, interruption, robot behaviours.

\section{INTRODUCTION}

Robots will become pervasive in our everyday environments. We will expect them to interact with people, and to do so in a socially acceptable manner. For robots to work in such social settings, both robot and human must understand each other's behaviours and respond accordingly. This is not yet something that we, as interaction designers, fully understand how to do. This leads to a two-part research question: how should a robot recognize and respond to human behaviours, and how should a robot behave so that it exhibits appropriate and understandable behaviour in a particular context?

One specific form of human-robot interaction that interests us concerns social interruption. There is already considerable past research on interruption and interruptibility in humancomputer interaction (HCI). McFarlane et al. identified different types and methods of interruptions as well as the ways humans respond to them, and the different phases that occur as the interruption is carried out. [1] Their models enable adapting a specific interruption to specific circumstances as determined by factors such as message urgency, the person's interruptibility state, etc. Other researchers have applied this theory to the design of contextually-aware desktop computer software that decides when and how to deliver information to its users. [2] As far as we know, there is still no robot behaviour model that directly addresses interruption.

In this report, we propose an early design approach to a human-robot interaction interruption model. Our discussion includes a critique of our design based on observations, done within our research group, of how a robot can interrupt a person and how the human interprets these actions. We believe the lessons learned through this critique will provide a better understanding of how to design future experiments.

Our critique is based on a series of Wizard of Oz studies where a robot is animated with particular interruption behaviours for a given context, and people are then asked to interpret that behaviour. We then check the accuracy of that interpretation. In this report, we contribute the methodology, design considerations and critique of such an interruption experiment. Our particular study was designed to explore a robot in a simplified interruption scenario, where the robot's behaviour suggests the urgency of the message it is trying to transmit. Our concern was how the human interprets these robot's actions. The actual experimental results are not our focus. Rather, we offer lessons learned via a critique, which should provide others with a better understanding of how to design similar experiments and should call for future research of interruption in human-robot interaction.

\section{DESCRIPTION OF THE EXPERIMENT}

Our study focus involved designing robot behaviours for social interruption. Fortunately, we could build upon the considerable research on human to human interruption. This past work identified the different types and methods of interruptions, the ways humans respond to them, and the different phases that occur as the interruption is carried out. (e.g., [1]) Specific interruption methods can thus be tailored to specific circumstances as determined by factors such as message urgency, the recipient's interruptibility state, and so on. Indeed, interruption theory has already been applied to the design of contextually-aware computer software that decides when and how to deliver information to its users. (e.g., [2]) Yet as far as we know, no research explicitly addresses robot behaviour when interrupting, or attempts to design an effective interruption model for human-robot interaction.

\section{A. Methodological Design - Identifying Behaviours}

Our first question is: can we identify appropriate behaviours that the robot could assume in particular situations? Our approach - which we believe is generalizable to other situations - was to identify appropriate human behaviour and mimic it as robotic behaviour. In particular, we identified five interruption scenarios, which in turn led to us scripting robot behaviours with varying levels of urgency and importance.

Three people - all members of our laboratory - were asked to act through various scenarios, where they had to interrupt two other people who were engaged in a meeting inside an office with an open door. Our scenario focused on the

Cite as:

Saulnier, P., Sharlin, E. and Greenberg, S. (2010) Exploring Interruption in HRI using Wizard of Oz. (Late Breaking Report). In DVD Proc. $5^{\text {th }}$ ACM/IEEE Int'l Conf on Human-Robot Interaction - HRI'2010, March 2-5, Osaka, Japan, 2 pages. 
interrupting people, who were constrained to show only the abilities that our target robot could replicate, i.e., motion, head movement, and proximity to the people being interrupted. They were not allowed to speak or to make sounds. We videotaped their actions, and identified characteristic behaviours, each designed to exhibit different levels of urgency.

\section{B. Methodological Design - Simulating Robot Behaviours}

Our second question is: can a human participant interpret the motions and intent (i.e., the social behaviour) of a robotic entity in an interruption scenario. To answer this, we scripted the previously identified behaviours so that could be followed by an operator. In a given interruption scenario, the operator would follow an algorithm - a script - to animate the robot to reflect a particular behaviour. This is an application of the Wizard of $\mathrm{Oz}(\mathrm{WoO})$ methodology, where an operator simulates computer actions (e.g., [3]) Participants were then expected to distinguish these robotic behaviours (e.g. behaviour A is "more urgent" than behaviour B.)

Each scenario involves a wheeled robot that approaches the participant working in a room. The participant was seated so that the doorway into the room was in their line of sight. Over time, a robot would approach and possibly enter the room through this doorway, where its behaviour could attract the attention and possibly interrupt the participant from his or her task. The specific behaviour of the WoO-controlled robot was chosen to convey varying levels of importance and urgency. During each interaction, the participant was encouraged to "think aloud", and then was subsequently asked to interpret the robot's behaviour and answer some questions. Our analysis would then determine which behaviour methods were easily understood and differentiated from other behaviours.

\section{IMPLEMENTATION}

A customized Pioneer-3DX robot capable of precise and robust locomotion was used as a base for the robot. We added a head attachment, which included a camera capable of moving with 3 degrees of freedom. We created custom software to wirelessly control the robot and the camera position via a video game controller. Status information, such as position and web cam imagery, was collected continuously from the robot and seen by the operator. Using the game controller, the operator animated the robot's behaviour by adjusting its motion and speed, and could simulate gaze and head gestures (such as nodding and head shaking) by moving the robot head.

\section{DESIGN CONSIDERATIONS AND CRITIQUE}

Designing a robot capable of executing various interruption behaviours via $\mathrm{WoO}$ proved non-trivial. Considerable ground work was required to create custom controller software that would operate the robot in all the ways required to create observable behaviour.

Safety is an important concern. In our case, the Pioneer$3 \mathrm{DX}$ robot has significant mass and could move at high speeds. Thus we equipped it with safety features to prevent it from running into people or other obstacles.
Executing pre-determined behaviours consistently is typical of Wizard of $\mathrm{Oz}$ [3]. Yet this was more difficult than anticipated due to variations in robotic actions and the environment, and imprecision in our behavioural specifications. Mimicking convincing behaviours via low-level control functions proved difficult for the operator to do in real time. Yet pre-coding behaviours as macros did not always give the same result (e.g., due to wheel slippage). Overall, the behaviours actually executed by the robot generally differed from the pre-planned, desired behaviours for each scenario, and the behaviour for each scenario varied slightly between each participant. On reflection, we believe we should have better definitions for the interruption behaviours (including acceptable variance), and a better balance between manual and automated control.

Suspension of disbelief. The phenomena being studied in these types of experiments implicitly assume that the social robotic entity has an internal purpose and intent driving its actions. Unless the human observer perceives the robot as a social entity, there is no reason for them to interpret the robots motions as anything more than abstract patterns of movement. It is critical to cultivate an atmosphere of open-mindedness and "suspension of disbelief" for the participants, where they are primed by instructions to make a "leap of faith" and associate the robot's motions with those social gestures they are already familiar with in daily life. Suddenly there is a "why" (which is the social/emotional message) behind the "what" of the robot's motions.

Study design. While we asked participants to interpret the robot's behaviour, we did not tell them if and how to act on that behaviour. Participants were thus confused as to how to interact with the robot following an interruption, and wondered why the robot did not react to their own actions. Better communication with the participant during the introduction may have avoided this problem.

Secondary effects. Our behaviours focused mainly on speed, motion, proximity and head gestures. Yet participants became aware of the robot's presence due to the sounds it made while moving, e.g., motor and wheel sounds, and the clicking noise emitted by the obstacle-detecting SONAR sensors. This introduced a confound that we should have considered as part of the robot's behaviour.

\section{FUTURE WORK}

In future work, we hope to explore a full user study that addresses the aforementioned problems. We plan to further understand how humans interpret robot interruption behaviour, and how the robot's abilities can be effectively enhanced.

\section{REFERENCES}

[1] McFarlane, D. C. and Latorella, K. A. 2002. The scope and importance of human interruption in human-computer interaction design. Hum.Comput. Interact. 17, 1 (Mar. 2002), 1-61.

[2] Fogarty, J. A. 2006 Constructing and Evaluating Sensor-Based Statistical Models of Human Interruptibility. Doctoral Thesis. UMI Order Number: AAI3241594., Carnegie Mellon University.

[3] Maulsby, D., Greenberg, S. and Mander, R. (1993) Prototyping an Intelligent Agent through Wizard of Oz. Proc ACM CHI, 277-284, May. 